\title{
Changes in Non-Invasive Wave Intensity Parameters with Variations of Savitzky-Golay Filter Settings
}

\author{
Nicola Pomella ${ }^{1}$, Mark Rakobowchuk ${ }^{2}$, Christina Kolyva ${ }^{3}$, Ashraf W Khir ${ }^{1}$ \\ ${ }^{1}$ Brunel University London, London, United Kingdom \\ ${ }^{2}$ Thompson Rivers University, Kamloops, Canada \\ ${ }^{3}$ Middlesex University London, London, United Kingdom
}

\begin{abstract}
Ultrasound-measured waveforms, such as vessel diameter and blood flow velocity, are used to perform analysis of waves in the cardiovascular system. Wave intensity analysis is one of the tools used for this purpose.

The waveforms are commonly filtered to eliminate high-frequency noise, however the filter settings affect the features of these signals and especially of their time derivatives, upon which wave intensity analysis is based.

This study aims to investigate the alterations of wave intensity parameters with varying Savitzky-Golay filter settings, one of the most common smoothing algorithms used in this context.

A broad spectrum of variations was observed in all the wave intensity variables. It is therefore important to always specify the filter settings applied to the signals in a wave intensity study, so that appropriate comparisons can be made.
\end{abstract}

\section{Introduction}

Wave Intensity Analysis (WIA) is a powerful tool developed by Parker and Jones [1] to study wave propagation in the cardiovascular tree and has been proved useful in the clinical setting [2]. The original formulation required invasive simultaneous measurements of blood pressure and flow velocity (U), but the parallel development of ultrasound systems enabled performing WIA through ultrasound-measured diameter (D) and U instead [3, 4].

$D$ and $U$ are commonly filtered (smoothed) to eliminate high-frequency noise; however the filter settings affect WIA parameters, calculated not directly from $\mathrm{D}$ and $\mathrm{U}$, but from their time derivatives (dD, dU) [5]. One of the most common smoothing algorithmsfor these signals is the Savitzky-Golay (SG) filter [6], which fits a sub-set of data points of the signal, contained in a window of specific length $(\mathrm{w})$, with a polynomial of a specific degree (p), via the least-squares method.

This study aims to investigate the alterations of WIA parameters with varying SG filter settings (i.e. $w$ and $p$ ).

\subsection{Wave intensity analysis}

Wave Intensity (dI) can be defined as the product $\mathrm{dD} \cdot \mathrm{dU}$ [4]. The calculation of local wave speed (PWV) (i.e. at the site of the measurement of $\mathrm{D}$ and $\mathrm{U}$ ) is performed through the lnDU-loop [4], assuming that there is no contribution of reflected waves to the pressure, diameter and velocity waveforms in early systole. Using the calculated PWV value one can separate dI into forward $\left(\mathrm{dI}_{+}\right)$and backward components (dI.) in so far as $\mathrm{dI}=\mathrm{dI}_{+}+\mathrm{dI}$.

Three waves can be assessed: the forward compression wave (FCW), generated by the contraction of the left ventricle, the backward compression wave (BCW), attributed to reflections from the downstream capillary bed, and the forward expansion wave (FEW), generated by the deceleration of the heart's contraction in late systole. The energy carried by each wave is calculated by the time integral of the corresponding wave.

\section{Methods}

\subsection{Instrumentation and measurements}

A SSD - 5500 ultrasound system (Aloka, Tokyo, Japan) equipped with a $7.5 \mathrm{MHz}$ linear array vascular probe was used to acquire single-beat $\mathrm{D}$ and $\mathrm{U}$ from the right common carotid artery (CCA) of a young, healthy individual (25 years) at rest in a supine position. The CCA was insonated $\sim 2 \mathrm{~cm}$ proximal to the bifurcation. D and $U$ were measured with a resolution of $0.013 \mathrm{~mm}$ and $0.012 \mathrm{~m} / \mathrm{s}$, respectively. The sampling frequency was $1000 \mathrm{~Hz}$. The D waveform was calculated as the distance 
between the two walls of the vessel over time. The gates were positioned manually between the media and the intima of the anterior and posterior walls, and parallel to them. The $U$ waveform was acquired ensuring that the Doppler gate was at the centre of the vessel, parallel to the walls, with an insonation angle equal to $60^{\circ}$.

\subsection{Data analysis}

Data analysis was performed via custom-made algorithms written in Matlab (version R2010b, The MathWorks, Inc., Natick, Massachusetts, USA). D and U were filtered with 42 SG settings, derived from combining 6 polynomial degrees p: 2-7, with 7 selected window lengths w: 9-21-33-45-71-99-119 points. PWV was calculated through the lnDU-loop and non-invasive WIA was performed [4]. PWV, maximum values, energies and durations of FCW $\left(\mathrm{FCW}_{\max }, \mathrm{FCW}_{\mathrm{e}}\right.$, $\left.\mathrm{FCW}_{\text {time }}\right), \mathrm{BCW}\left(\mathrm{BCW}_{\max }, \mathrm{BCW}_{\mathrm{e}}, \mathrm{BCW}_{\text {time }}\right), \mathrm{FEW}$ $\left(\mathrm{FEW}_{\text {max }}, \mathrm{FEW}_{\mathrm{e}}, \mathrm{FEW}_{\text {time }}\right)$ were compared between the 42 settings.

\section{Results}

PWV increased with increasing $\mathrm{w}$ and decreasing $\mathrm{p}$ ( $12 \%$ at $\mathrm{p}=2$ between $\mathrm{w}=9$ and $\mathrm{w}=119,6 \%$ at $\mathrm{p}=7$ in the same window range). $\mathrm{FCW}_{\text {time, }} \mathrm{BCW}_{\text {time }}$ and $\mathrm{FEW}_{\text {time }}$ increased with w and decreased with p (25\%, 100\%, 80\% at $p=2$ between $w=9$ and $w=119$, respectively, and $60 \%$, $40 \%, 25 \%$ at $\mathrm{p}=7$ ). The maximum variation along $\mathrm{p}$ was $22 \%, 32 \%$ and $59 \%$ for $\mathrm{FCW}_{\text {time }}, \mathrm{BCW}_{\text {time }}$ and $\mathrm{FEW}_{\text {time, }}$, respectively.

Variations were much greater for peak and energy values. $\mathrm{FCW}_{\max }$ (Fig. 1) and $\mathrm{FEW}_{\max }$ decreased with increasing $\mathrm{w}$ and decreasing $\mathrm{p}$ (up to 4-fold at $\mathrm{p}=2$, up to 4-fold at $p=7$ for $\mathrm{FCW}_{\max }$; up to 4-fold at $\mathrm{p}=2$, up to 43fold at $\mathrm{p}=7$ for $\left.\mathrm{FEW}_{\max }\right)$. Although the pattern of changes is similar for $\mathrm{FCW}_{\max }$ and $\mathrm{FEW}_{\max }$, the latter exhibited an unexpected huge gap between $\mathrm{w}=9$ and $\mathrm{w}=21$, the two shortest window lengths, for $\mathrm{p}=3-7$.

In fact, the variation between the two shortest windows was about 8 -fold at $\mathrm{p}=7$, and only about 4 -fold between $\mathrm{w}=21$ and $\mathrm{w}=119$ at the same p. The combined overall gap is therefore around 43-fold. The maximum variation along $\mathrm{p}$ was $100 \%$ for $\mathrm{FCW}_{\max }$ and 9 -fold for $\mathrm{FEW}_{\max }$. If $\mathrm{w}=9$ had been excluded, the variation would have lowered to 2.25-fold for $\mathrm{FEW}_{\text {max. }}$

$\mathrm{BCW}_{\max }$ decreased with $\mathrm{w}$ (up to 81-fold at $\mathrm{p}=2$, up to 7-fold at all other values of $p$ ) and decreased with $p$ up to 14-fold, only excluding $\mathrm{p}=6$ and $\mathrm{p}=7$, which would have brought the variation up to 1040 -fold. In fact, the maximum change between $\mathrm{p}=2$ and $\mathrm{p}=5$ is about 14 -fold while the maximum change between $p=5$ and $p=7$ is about 75-fold. Too high polynomial degrees caused an unrealistic variation. Also in the case of $\mathrm{BCW}_{\max }, \mathrm{w}=9$ presented some unrealistic values.

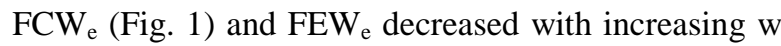
and decreasing $\mathrm{p}\left(100 \%\right.$ at $\mathrm{p}=2$ and $8 \%$ at $\mathrm{p}=7$ for $\mathrm{FCW}_{\mathrm{e}}$, 1.6-fold at $p=2$ and 9-fold at $p=7$ for $F E W_{e}$ ). The maximum variation along $\mathrm{p}$ was $100 \%$ and 6 -fold for $\mathrm{FCW}_{\mathrm{e}}$ and $\mathrm{BCW}_{\mathrm{e}}$, respectively. In contrast, $\mathrm{BCW}_{\mathrm{e}}$ increased with increasing $\mathrm{w}$ up to $\mathrm{p}=4$ (about 2.5-fold at $p=2-4$ ) and decreased for high $p$ (up to $100 \%$ at $p=7$ ). The maximum variation along p was 5.6-fold.

Finally, a general shift of the signal towards increasing time values can be seen with increasing w. Large window lengths cause a significant truncation of the signal and some early-systolic features could be lost (Fig. 2).

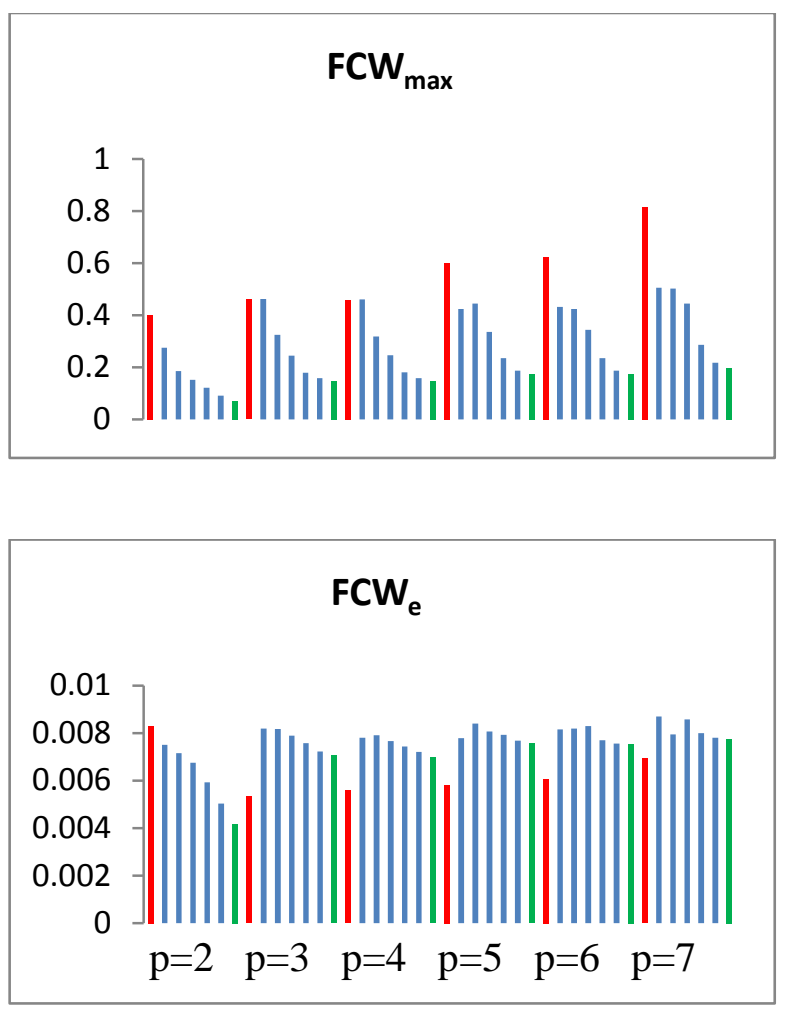

Figure 1.Variations of FCW $\max$ (top) and $\mathrm{FCW}_{\mathrm{e}}$ (bottom) with changes of Savitzky-Golay filter settings. The value of $p$ (polynomial degree) is indicated in the horizontal axis. For each p, columns represent different increasing $\mathrm{w}$ values (window lengths): 9-21-33-45-71-99-119 points. In particular, the red column stands for $\mathrm{w}=9$ and the green column for $w=119$. Units of $\mathrm{FCW}_{\max }$ and $\mathrm{FCW}_{\mathrm{e}}$ are of $\mathrm{mm}^{2} / \mathrm{s}$ and $\mathrm{mm}^{2}$, respectively.

\section{Discussion}

WIA parameters are highly affected by the change of SG filter settings, showing a broad spectrum of variation. The correct choice of $\mathrm{w}$ is critical, as it affects the signal more than $\mathrm{p}$. 
Energy parameters $\left(\mathrm{FCW}_{\mathrm{e}}, \mathrm{BCW}_{\mathrm{e}}, \mathrm{FEW}_{\mathrm{e}}\right)$ appeared to be less sensitive to the filter settings than their corresponding intensities $\left(\mathrm{FCW}_{\max }, \mathrm{BCW}_{\max }, \mathrm{FEW}_{\max }\right)$. This is likely due to the way these variables are computed: peaks involve the detection of single points, while areas involve an integral which takes account of a number of points. FCW values, compared to the other energy values, are mostly affected by the truncation of the signal, especially at large windowlengths. This phenomenon in fact causes loss of information during the early systolic period.

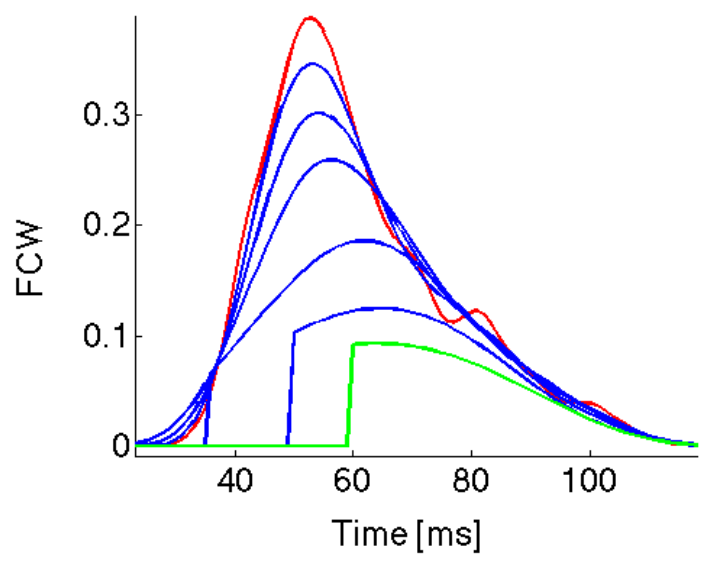

Figure 2. Variation of FCWwith the window length w, at a constant polynomial degree $\mathrm{p}=2$. Red line corresponds to $\mathrm{w}=9$ and green line to $\mathrm{w}=119$. Intermediate values $\mathrm{w}=21-33-45-71-99$ are represented in blue. The decreasing pattern of $\mathrm{FCW}_{\max }$ with increasing $\mathrm{w}$ is clearly visible, as well as the shift of the signal to the right. Noise can be seen on the red curve, while at large window lengths the signal is truncated $(\mathrm{w} \geq 71)$. Units are of $\mathrm{mm}^{2} / \mathrm{s}$.

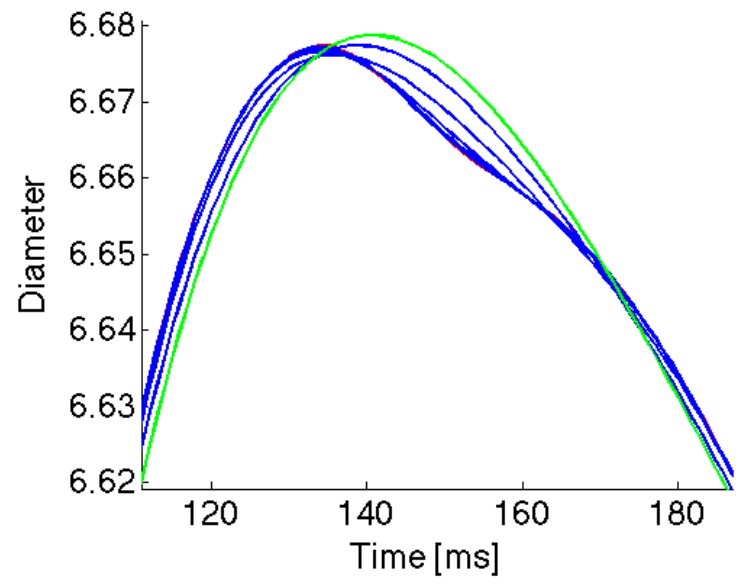

Figure 3. Variation of the maximum value of $\mathrm{D}$ with the window length $w$, at a constant polynomial degree $\mathrm{p}=2$. Red line corresponds to $\mathrm{w}=9$ and green line to $\mathrm{w}=119$. Intermediate values $\mathrm{w}=21-33-45-71-99$ are represented in blue. The truncation of the signals at high $\mathrm{w}$ is not visible, as it happens at earlier time points. Physiological features of the waveform are lost at high w (see the green line). The overall variation of maximum value of $\mathrm{D}$ with window length is not significant $(<0.013 \mathrm{~mm}$, the resolution of the ultrasound machine). Units of $\mathrm{D}$ are of $\mathrm{mm}$.

BCW seemed the most affected among the main three physiological waves by the change of filter settings. Too high a polynomial degree or too short a window caused unrealistic values. This is likely due to the fact that small windows and high polynomial degrees tend to preserve the high-frequency noise producing artifacts. In contrast, large windows and low polynomial degrees tend to dampen natural variations of the waves and of the original signals (Fig. 2-3).

\section{Conclusions}

Ultrasound-measured vessel diameter and blood flow velocity waveforms were not significantly affected by the Savitzky-Golay filter settings (the variations of the peak of $\mathrm{D}$ are depicted in Fig. 3) but their time derivatives $\mathrm{dD}, \mathrm{dU}$ and wave intensity parameters showed a broad spectrum of variation with changes of filter settings.

We recommend to always specify the SavitzkyGolay filter settings applied to the signals in a wave intensity study, so that appropriate comparisons can be made between studies.

The present findings hold for waveforms that were sampled at $1000 \mathrm{~Hz}$. Different sampling frequencies could produce different results.

\section{Acknowledgements}

The authors would like to thank E. N. Wilhelm and J. González-Alonso for their technical support with the ultrasound measurements. The authors would also like to thank the participant in this study.

\section{References}

[1] Parker KH, Jones CJ. Forward and backward running waves in the arteries: analysis using the method of characteristics. J BiomechEng 1990; 322:326-112(3).

[2] Jones CJ, Parker KH, Hughes R, Sheridan DJ. Nonlinearity 
of human arterial pulse wave transmission. J BiomechEng 1992; 10:14-114(1).

[3] Niki K, Sugawara M, Uchida K, Tanaka R, Tanimoto K, Imamura $\mathrm{H}$, Sakomura $\mathrm{Y}$, Ishizuka $\mathrm{N}$, Koyanagi $\mathrm{H}$, Kasanuki H. A noninvasive method of measuring wave intensity, a new hemodynamic index: application to the carotid artery in patients with mitral regurgitation before and after surgery. Heart Vessels 1999; 263:271-14(6).

[4] Feng J, Khir AW. Determination of wave speed and wave separation in the arteries using diameter and velocity. J Biomech 2010; 455:462-43(3).

[5] Rivolo S, Asrress KN, Chiribiri A, Sammut E, Wesolowski R, Bloch LØ, Grøndal AK, Hønge JL, Kim WY, Marber M, Redwood S. Enhancing coronary Wave Intensity Analysis robustness by high order central finite differences. Artery Res 2014; 98:109-8(3).

[6] Savitzky A, Golay MJ. Smoothing and differentiation of data by simplified least squares procedures. Anal Chem 1964; 1627:1639-36(8).

Address for correspondence.

Ashraf W. Khir.

Institute of Environment, Health and Societies - Biomedical Engineering Research Theme, Brunel University London, Kingston Lane, Uxbridge, Middlesex, UB8 3PH, United Kingdom. Email address: Ashraf.khir@brunel.ac.uk 